\title{
Blood glucose concentration compared in EDTA/F plasma and serum in a referral clinical laboratory in Nepal
}

Vivek Pant ${ }^{1}$, Keyoor Gautam², Santosh Pradhan ${ }^{1}$, Devish Pyakurel ${ }^{2}$, Abha Shrestha ${ }^{2}$

${ }^{1}$ Department of Clinical Biochemistry, Samyak Diagnostic; Jawalakhel, Lalitpur, Nepal

${ }^{2}$ Department of Pathology, Samyak Diagnostic; Jawalakhel, Lalitpur, Nepal

Keywords:
Glucose;
Plasma;
Serum;
Serum separator tubes;
Sodium fluoride tubes;

\section{Correspondence:}

Dr. Vivek Pant, $M D$

Department of Clinical Biochemistry,

Samyak Diagnostic; Jawalakhel, Lalitpur, Nepal

ORCID ID: 0000-0002-3967-1851

Email:drvpant@gmail.com

Reveived : October $20^{\text {th }} 2020$; Accepted : November $22^{\text {nd }} 2020$ significant.
Citation : Pant V, Gautam K, Pradhan S, Pyakurel D, Shrestha A. Blood glucose concentration compared in EDTA/F plasma and serum in a referral clinical laboratory in Nepal. J Pathol Nep. 2021;11: 1837-41. DOI: 10.3126/jpn.v10i2.32351

Copyright: This is an open-access article distributed under the terms of the Creative Commons Attribution 4.0 International License, which permits unrestricted use, distribution, and reproduction in any medium, provided the original author and source are credited.
Background: Sodium fluoride tubes or serum separator tubes are mostly used for blood glucose estimation in the clinical laboratories of Nepal. The study aimed to investigate the stability of glucose in samples collected in serum separator tubes and sodium fluoride/sodium ethylenediaminetetraacetic tubes simulate prolonged sample transport between venipunctures, centrifugation, and measurement.

Materials and Methods: Samples were collected from healthy volunteers into two different serum separator tubes and two different sodium fluoride/sodium ethylenediaminetetraacetic tubes. Glucose concentration was measured at 30 minutes after venipuncture and compared with results from the same samples analyzed at four hours and with the results from tubes centrifuged with a delay of 4 hours. Results: When comparing plasma glucose at 30 minutes, glycolysis caused a relative reduction of the glucose concentration in serum at 30 minutes of $3.1 \%$, which is only slightly less than at 4 hours (3.7 \%). This is still substantially more than the reduction in plasma at 4 hours $(1.3 \%)$. Surprisingly, the difference between plasma glucose at 30 minutes and serum glucose at 4 hours was only $1.9 \%$ which is not clinically

Conclusions: The Na-F/Na2 EDTA tubes and serum separator tubes can be used interchangeably for analysis of blood glucose up to 4 hours if centrifuged within 30 minutes.

\section{INTRODUCTION}

Glucose can be measured in whole blood, serum(S-Glucose), or plasma (P-Glucose), but plasma is recommended for diagnosis. ${ }^{1}$ Loss of glucose in collection tubes by glycolysis is a serious problem and is often overlooked in our settings. There are a number of enzyme inhibitor/anticoagulant combinations that are available for the stabilization of glucose in the collection tube. The most commonly used glycolysis inhibitor is sodium fluoride $(\mathrm{NaF})$, which inhibits the enzyme enolase. NaF can be used alone or with anticoagulants such as potassium oxalate, ethylenediaminetetraacetic (EDTA), citrate, or lithium heparin. The recent recommendation for stabilization of blood glucose is the use of tubes containing 
a rapid glycolysis inhibitor, i.e. citrate/EDTA buffer which inhibits the upstream enzymes involved in glycolysis unlike the $\mathrm{NaF}$ which inhibits the downstream enzyme and allows glycolysis to continue for a considerable time. ${ }^{1,2}$ However, the choice and use of collection tubes in the clinical laboratory also depends upon the availability and economic aspects.

The original recommendations of the American Diabetes Association (ADA) for the stabilization of blood glucose, indicated the following: (1) immediate centrifugation and separation of plasma from blood cells, (2) immediate cooling of the sample tube in an ice-water slurry, and plasma separation within $30 \mathrm{~min}$ from blood draw. Clinical organizations do not recommend the measurement of S-Glucose rather than P-Glucose for the diagnosis of diabetes. ${ }^{3,4}$ In Nepal, the newly recommended citrate buffer, $\mathrm{NaF}$ and EDTA (acidified mixture) containing tubes are costlier and are unavailable. Thus, we are left with the option of using $\mathrm{NaF}$ tubes with EDTA or potassium oxalate and centrifuging the sample immediately for separation of plasma or relying on serum separated under carefully selected conditions.

Serum separator tubes (SST) have been extensively used by the laboratories in Nepal for the estimation of S-Glucose. During the centrifugation process, the separator gel in these tubes liquefies and migrates to form a physical barrier between the serum and the cellular components thus preventing glycolysis by the red blood cells. Various studies have shown that there was no difference in glucose concentrations of blood samples collected in SST and fluoride tubes that were separated within two hours of collection. ${ }^{5,6,7}$

At our laboratory, we are using sodium fluoride/sodium EDTA (NaF/Na2EDTA) tube for the estimation of P-Glucose. There is no uniformity in the use of blood collection tubes in clinical laboratories in Nepal. The purpose of this study is to investigate the stability of glucose in samples collected in SST and $\mathrm{NaF} / \mathrm{Na} 2 \mathrm{EDTA}$ tubes by comparing the glucose concentration at 30 minutes and 4 hours, collected and handled differently. The estimation of sample glucose in different tubes at a different times from the same sample will help us identify the difference in the result which ultimately guides the clinician.

\section{MATERIALS AND METHODS}

A cross-sectional observational study was performed at the Samyak Diagnostic Pvt Ltd Clinical Laboratory based in Lalitpur, Kathmandu. Blood samples for glucose concentration measurements were collected from sixtythree participants irrespective of their age, gender, fastingand disease state. Written informed consent was obtained from all participants. Ethical approval to conduct this study was obtained from the Nepal Health Research Council (Protocol number- 932/2019 P).

Different glucose measurements were done for each participant on four occasions based on samples obtained at the same time but collected and handled differently. For this, total of $8 \mathrm{ml}$ of venous blood was drawn in two $\mathrm{NaF} /$ Na2EDTA tubes for plasma (P1 and P2) and two serum separator tubes for serum (S1 and S2).

All venipunctures were performed by a single experienced phlebotomist to minimize venipuncture bias and were carried out between $7 \mathrm{am}$ and $10 \mathrm{am}$. The room temperature during blood collection was $26.0^{\circ} \mathrm{C}$ (range $23.6-28.6^{\circ} \mathrm{C}$ ). Visibly lipemic, icteric or hemolyzed samples were not included in the study. The plasma tubes used were BD Vacutainer sodium fluoride/sodium EDTA $13 \times 75 \mathrm{~mm}, 2$ $\mathrm{ml}$ and the serum tubes used were BD Vacutainer serum separator tube (SST) $13 \times 100 \mathrm{~mm}, 5 \mathrm{ml}$.

P1 and S1 were allowed to clot for 20 minutes and then centrifuged at $1600 \times \mathrm{g}$ for $10 \mathrm{~min}$ using the Remi Neya 4 (Remi Elektrotechnik Ltd, India) centrifuge. Then the glucose concentration was determined (results presented as P1a and S1a) at $30 \mathrm{~min}$ after blood collection. The remaining plasma and serum were stored at $4{ }^{\circ} \mathrm{C}$. The glucose concentration was determined again after four hours after bringing the tubes at room temperature (P1b and $\mathrm{S} 1 \mathrm{~b}$ ). The $\mathrm{P} 1 \mathrm{~b}$ and $\mathrm{S} 1 \mathrm{~b}$ tubes are mentioned as 4 hours tubes with early centrifugation. P2 and S2 were allowed to clot for four hours at room temperature and then centrifuged after which the P-glucose and S-glucose, respectively were determined (P2a and S2a). The P2a and S2a tubes are mentioned as 4 hours tubes with delayed centrifugation. The samples were collected in the phlebotomy room of this laboratory. Therefore, there was no delay in transportation and no effect on environmental temperature.

The glucose concentration was determined spectrophotometrically using Randox Imola auto-analyzer (Randox Laboratories Limited UK) by a glucose oxidaseperoxidase method. The quality control of the measurements used two levels of quality control material and conducted by the qualified technical personnel and properly documented. Paired specimens (both serum and plasma) from each participant were analyzed using the same lot of reagent, eliminating any lot-to-lot variability in the results. The same technician performed the measurements of all study specimens.

All data sets were tested for normality using the KolmogorovSmirnov test and expressed as mean \pm standard deviation (SD) if normally distributed and as median if not normally distributed. The comparison was evaluated in a pairwise model, i.e. the Student's dependent test and the Wilcoxon rank test, as appropriate. Deming regression was used to 
Table 1: Mean blood glucose concentration in various tubes at different times along with percentage decrease in mean blood glucose (when P1a is taken as the reference) and the level of significance among various tubes

\begin{tabular}{lcc}
\hline $\begin{array}{c}\text { Variable } \\
{[\text { Mean } \pm \text { Standard deviation }(\mathbf{m g} / \mathbf{d L})]}\end{array}$ & Percentage decrease in mean blood glucose (\%) & p-value (Wilcoxon signed rank) \\
\hline $\begin{array}{l}\text { S1a versus P1a } \\
{[\mathbf{9 7 . 7} \pm \mathbf{1 4 . 8} \text { versus } \mathbf{1 0 0 . 8} \pm \mathbf{1 6 . 6}]}\end{array}$ & 3.07 & $<0.001$ \\
\hline $\begin{array}{l}\text { P1b versus P1a } \\
{[\mathbf{9 9 . 5} \pm \mathbf{1 7} \text { versus } \mathbf{1 0 0 . 8} \pm \mathbf{1 6 . 6}]}\end{array}$ & 1.28 & $<0.001$ \\
\hline $\begin{array}{l}\text { P2a versus P1a } \\
{[\mathbf{9 3 . 5} \pm \mathbf{1 7} \text { versus } \mathbf{1 0 0 . 8} \pm \mathbf{1 6 . 6}]}\end{array}$ & 7.24 & $<0.001$ \\
\hline $\begin{array}{l}\text { S1b versus P1a } \\
{[\mathbf{9 7 . 0} \pm \mathbf{1 5} \text { versus } \mathbf{1 0 0 . 8} \pm \mathbf{1 6 . 6}]}\end{array}$ & 3.70 & $<0.001$ \\
\hline S2a versus P1a & 18.05 & $<0.001$ \\
\hline $\mathbf{8 2 . 6} \pm \mathbf{1 8 . 5}$ versus $\mathbf{1 0 0 . 8} \pm \mathbf{1 6 . 6}]$ &
\end{tabular}

$* P 1 a=$ Sodium fluoride tube at $30 \mathrm{~min}$, S1 $a=$ Serum tube at $30 \mathrm{~min}$, P1b =Sodium fluoride tube at 4 hours with early centrifugation, S1b = Serum tube at 4 hours with early centrifugation, $P 2 a=$ Sodium fluoride tube at 4 hours with delayed centrifugation, $S 2 a=$ Serum tube at 4 hours with delayed centrifugation

Table 2: Deming regression functions and Pearson correlation coefficient (r)

\begin{tabular}{lccc}
\hline \multicolumn{1}{c}{ Variable } & Deming Slope \pm Sd $(\mathbf{m g} / \mathbf{d L})$ & Intercept \pm Sd & R \\
\hline P1b versus P1a & $1.02 \pm 0.01$ & $-3.37 \pm 1.46$ & 0.99 \\
\hline S1b versus P1a & $0.90 \pm 0.03$ & $6.12 \pm 2.85$ & 0.97 \\
\hline S2a versus P1a & $1.12 \pm 0.05$ & $-29.95 \pm 4.59$ & 0.95 \\
\hline
\end{tabular}

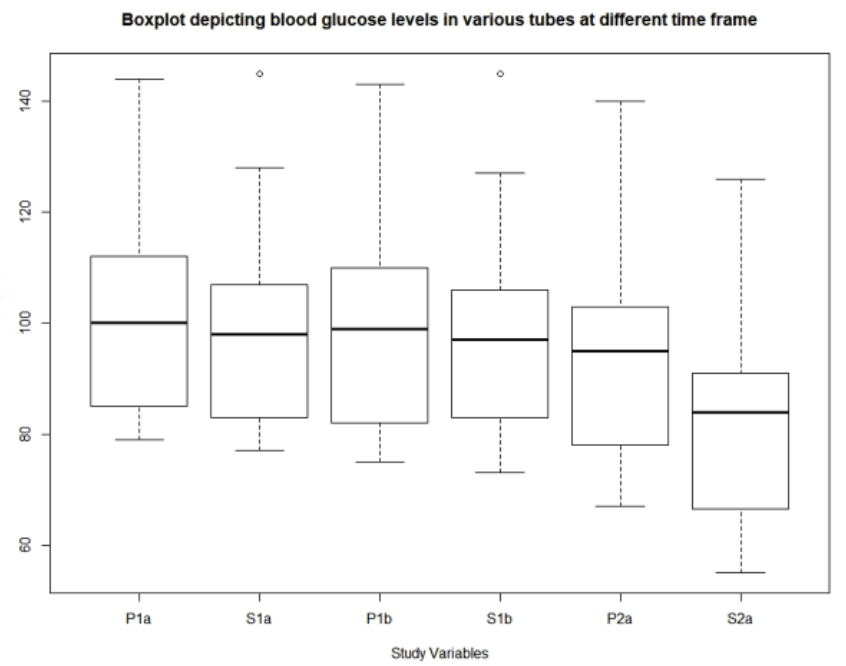

Figure 1: Mean P/S-Glucose at different times

compare glucose concentrations in $\mathrm{NaF} / \mathrm{Na} 2 \mathrm{EDTA}$ tubes and SST assuming the same method uncertainty (variance) for the compared measurements.

The statistical analysis was performed with Statistical Package for Social Sciences (SPSS v.23). The regression analysis for method comparison was done using the Association of Clinical Biochemistry and Laboratory Medicine (ACB) spreadsheet for verification and method/ patient comparison. ${ }^{89}$ Values of $\mathrm{p}<0.05$ were considered statistically significant.

There is a large amount of information in comparisons of sets of data that are not always explored. The ACB spreadsheet

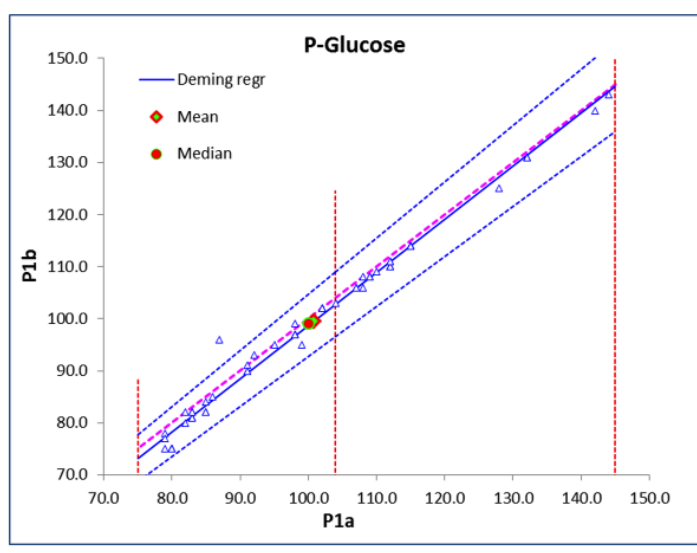

Figure 2- Deming regression of P-Glucose concentrations at 30 minutes (P1a) and four hours (P1b). The solid line is the regression line and the hatched line is the equal line. The two hatched lines forming a funnel-like entity delineate the allowable error zone, in this case the regression $\pm 6 \%$. Vertical lines indicate the measurement interval and reference value (104 mg/ $d L)$. The average and median values of the cohort are marked.

automatically presents regression and clinical performance from many different angles, allows the operator to partition the dataset, and presents the Bland-Altman ${ }^{10}$ difference graphs in absolute and relative terms.

\section{RESULTS}

The P-Glucose and S-Glucose decreased with time, faster in serum than in plasma (fig. 1). The mean glucose concentration at 30 minutes, 4 hours with early centrifugation, and 4 


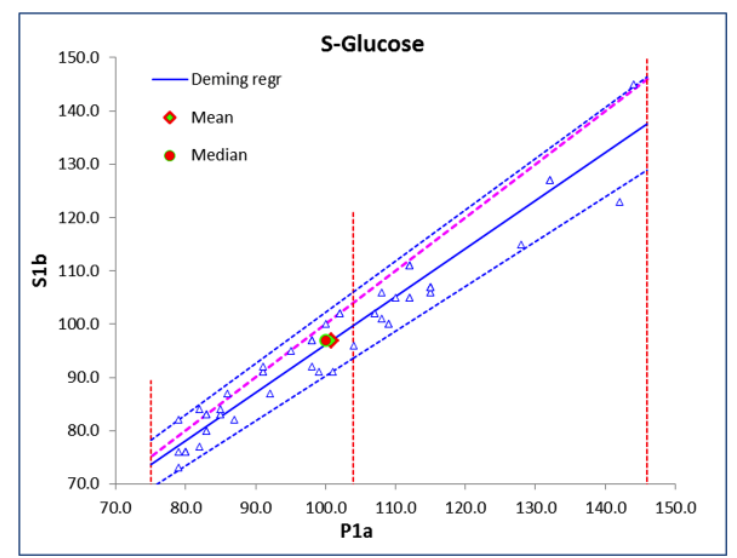

Figure 3: Deming regression of S-Glucose and P-Glucose concentrations in samples from the same blood draw at 30 minutes (P1a) and four hours (S1b). Symbols as in figure 2.

hours with delayed centrifugation along with the percentage decrease in mean blood glucose concentration and the level of significance in between tubes are shown in table 1 .

The plasma samples at 30 minutes $(\mathrm{P} 1 \mathrm{a})$ were taken as the reference method and were compared with $\mathrm{P} 1 \mathrm{~b}$ (fig. 2), S1b (fig 3), and S2a (fig, 4) (table 2). An allowable error zone of $\pm 6 \%$ around the Deming regression line was calculated and shown as "funnel-shaped" areas (fig. 2 to 4). The zones included $98 \%, 98 \%$, and $63 \%$ of the sample results, in the comparison of P1b, S1b, and S2a to P1a, respectively.

\section{DISCUSSION}

In the present study, we found that once the serum is separated from the red cells and analyzed within 30 minutes of collection, the glucose concentration will be stable for at least 4 hours and will be the same as in plasma stored under the same conditions. Though the difference at 30 minutes and four hours was statistically significant, this difference was within $\pm 6 \mathrm{mg} / \mathrm{dL}$, which is within the standard acceptable range as defined by the United States Clinical Laboratory Improvement Amendments (CLIA) guideline. ${ }^{11}$

When centrifugation was delayed for 4 hours, there was a large difference in the glucose concentration between serum and plasma samples. We considered 4 hours delay because we receive blood samples from various collection sites and private clinics and transportation may take considerable time. Sometimes the received samples do not come centrifuged. The difference between early centrifuged (P1a and S1a) and delayed centrifuged (P2a and S2a) samples was significant both statistically and clinically in both plasma and serum, i.e. $> \pm 6 \mathrm{mg} / \mathrm{dl}$. The relative mean decrease was $7.2 \%$ and $18.1 \%$ in delayed centrifuged plasma and serum samples, respectively (Table 1). A similar finding has been reported by the earlier studies. ${ }^{6,7}$

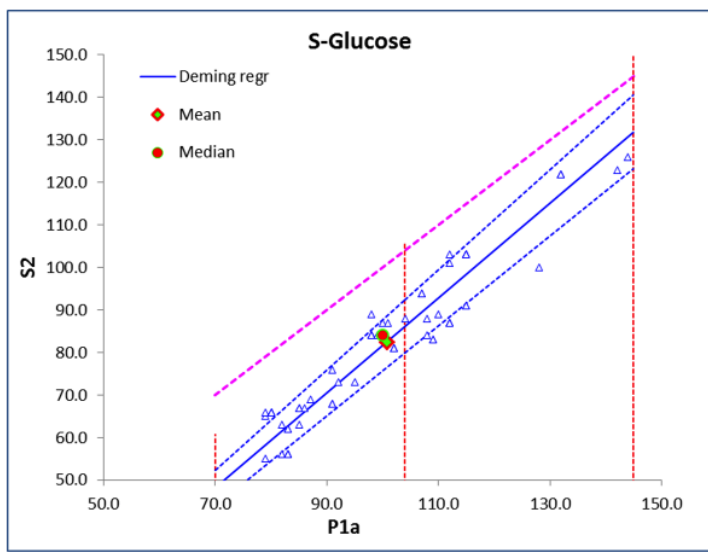

Figure 4: Deming regression of S-Glucose and P-Glucose concentrations in samples from the same blood draw at 30 minutes (Pla) and four hours (S2a). Symbols as in figure 2.

It is clear that the laboratories using $\mathrm{NaF} / \mathrm{Na} 2 \mathrm{EDTA}$ tubes will report slightly higher blood glucose than the laboratories using serum tubes. In our study, when samples from $\mathrm{NaF} / \mathrm{Na} 2 \mathrm{EDTA}$ tubes at 30 minutes (P1a) were taken as the reference, the glycolysis caused a relative reduction of the S-Glucose at 30 minutes (S1a) of $3.1 \%$ and four hours (S1b) of $3.7 \%$. However, the glycolysis caused a reduction in P-Glucose at four hours (P1b) of only $1.3 \%$.

The Deming regression of $\mathrm{P} 1 \mathrm{~b}$ compared with $\mathrm{P} 1 \mathrm{a}$ as the reference, showed a regression coefficient of about 1.02 and an intercept of about $3 \mathrm{mg} / \mathrm{dL}$ (Table 2). The correlation coefficient (r) was 0.99. In a comparison between S1b and P1a the slope was 0.90 and the intercept was $6 \mathrm{mg} / \mathrm{dL}$ with a correlation coefficient of 0.97 . Within the measuring interval, this corresponds to a mean difference of $1.9 \%$ which is not clinically significant. Thus, the $\mathrm{NaF} / \mathrm{Na} 2 \mathrm{EDTA}$ and serum tubes can be used interchangeably for analysis of blood glucose up to four hours if centrifuged at 30 minutes.

The regression analysis of $\mathrm{NaF}$ tubes at $30 \mathrm{~min}$ (P1a) versus SST at four hours with delayed centrifugation (S2a) (fig 4) demonstrated that there is a considerable decrease in blood glucose of $18.1 \%$ in S2a but the correlation coefficient ( $\mathrm{r}=$ 0.95 ) is acceptable (Table 2).

Statistical and clinical significance may be different quantities, where the latter is related to a difference between two results which might trigger a change in the patient care. For P/S-Glucose this amounts to $\pm 6 \%$ (CLIA) 11 and if shown in a diagram appears as a funnel-like zone surrounding the regression (fig 2, 3, 4). The comparison between P1b and P1a and S1b and P1a showed that $98 \%$ of the results would be within the zone whereas the corresponding result of the comparison between S2 and P1a was about $63 \%$. If, however, the allowable error zone was $10 \%$ then $98 \%$ of the results would be found within the zone. 
The minimal significant difference can also be calculated as the "MD=minimal difference" or the "RCV = reference change value" 12 using the formulas

$\mathrm{MD}=\mathrm{k} \times \sqrt{ }(\mathrm{uA} 2+\mathrm{uB} 2)$ and $\mathrm{RCV}=\mathrm{k} \times \sqrt{ }(\mathrm{uA} 2+\mathrm{uB} 2+2 \times \mathrm{uBio} 2)$

Where $\mathrm{k}$ is the coverage factor (conventionally set to 2 to cover $95 \%$ of the variation) and $\mathrm{uA}, \mathrm{uB}$ and $\mathrm{uBio}$ represent the uncertainty of the first, second, and within-individual biological variation, respectively.

\section{CONCLUSIONS}

Blood glucose measurement in serum can be accepted if the serum separation via centrifugation is achieved within 30 minutes. The avoidance of an extra amount of blood draw in a separate $\mathrm{NaF}$ tube and better turn-around-time are advantages of using serum tubes. It should be mandatory to have a centrifuge at all collection centers to achieve blood separation within a reasonable time.

\section{Footnote}

In this report, IFCC-IUPAC notation for measurands was used. Thus, P-glucose stands for plasma glucose concentration and S-glucose stands for serum glucose concentration. ${ }^{13}$

\section{Acknowledgment}

Authors would like to thank Dr. Anders Kallner affiliated with the Department of Laboratory Medicine, Karolinska Institute, Sweden for his very generous contribution in the preparation of this manuscript.

\section{Conflict of interest: None}

\section{REFERENCES}

1. Sacks DB,Arnold M, Bakris GLet al. Guidelines and recommendations for laboratory analysis in the diagnosis and management of diabetes mellitus. Clin chem 2011;57:e1-47. Crossref

2. Bonetti G, Carta M, Lapolla A et al. Correct determination of glycemia in the diagnosis and management of diabetes: Recommendations for the optimization of the pre-analytical phase. Nutr Metab Cardiovasc Dis. 2019;29:1-3. Crossref

3. American Diabetes Association. Standards of medical care in diabetes-2010. Diabetes care 2010;33:S11-61. Crossref

4. World Health Organization, 2006. Definition and diagnosis of diabetes mellitus and intermediate hyperglycaemia: report of a WHO/IDF consultation. Website

5. Fernandez L, Jee P, Klein MJ et al. A comparison of glucose concentration in paired specimens collected in serum separator and fluoride/potassium oxalate blood collection tubes under survey 'field' conditions. Clin biochem 2013;46:285-8. Crossref

6. Bruns DE. Are fluoride-containing blood tubes still needed for glucose testing? Clin biochem 2013;46:289-90. Crossref

7. Bhargava, M., Singh, N.P. \& Gupta, A.K. Should we still collect blood glucose sampling in fluoride tubes? An evidence-based study. Int J Diabetes Dev Ctries 2019;39:243-244. Crossref
8. 2018-07 acb method comparison patient samples. Accessed on: 01/09/2020. Website

9. Kallner A. Comprehensive method comparisons: getting more from the data. Accreditation and Quality Assurance 2014;9:451-7. Crossref

10. Bland JM, Altman DG. Statistical methods for assessing agreement between two methods of clinical measurement. Lancet 1986;1:30710. Website

11. Harris EK, Yasaka T. On the calculation of a" reference change" for comparing two consecutive measurements. Clin Chem 1983;29:2530. Website

12. Olesen HE, Ibsen I, Bruunshuus I et al. Properties and units in the clinical laboratory sciences. X. Properties and units in general clinical chemistry (Technical Report) (IFCC-IUPAC 1999). Pure and Applied Chemistry 2000;72:747-972. Website 